\title{
La nasalisation du déterminant en contexte non nasal en créole haïtien : un fait de langues résultant de l'analogie
}

\section{David TÉZIL}

Indiana University

\section{Résumé}

Cette étude s'intéresse au rôle de l'analogie dans les aspects sociolinguistiques et cognitifs d'un changement morphophonologique en cours en créole haïtien. Précisément, il s'agit de la nasalisation hors contexte nasal du déterminant postposé. Dejean (1980) et Joseph (1984) avaient remarqué ce qu'ils considéraient comme une variation libre entre, par exemple chat la et chat lan «le chat». Valdman (1991) a entrepris une étude pilote qui indiquait que cette variation était un changement en cours conduit par des locuteurs bilingues urbains jeunes. Dans cette même étude que nous avons reprise en 2014 à plus grande échelle et avec plus de rigueur avec toutes classes de sujets (urbains ou ruraux, mono ou bilingues), nous avons pu constater que ce changement a été provoqué à partir du modèle dûment décrit par la notion de l'analogie de Saussure (1916), c'est-à-dire par association et/ou par recherche de similitudes aux syntagmes comme jenou an «le genou » et pitimi an «le millet». Toutefois, même si la nasalisation du déterminant s'est généralisée dans presque tous les contextes (ex. diri an «le riz », pè an «le prêtre», mizik lan « la musique»), il y existe encore une certaine résistance au niveau des voyelles basses (ex. papa $a{ }^{*}$ *an « le père », anana $a / * a n$ « l'ananas »). Cette résistance s'explique en raison d'économie articulatoire.

\section{Rezime}

Etid sa a se yon ankèt ki fèt sou wòl analoji nan aspè sosyolinguistik ak konitif ke nou konstate nan yon chanjman mòfofonolojik $\mathrm{k}$ ap devlope nan lang kreyòl ayisyen an. Pou pi presizeman, sou fenomèn nazalizasyon detèminan apre yon fonèm oral (osinon kontèks non nazal). Yves Dejean (1980) ak Yves Joseph (1984) se de moun ki kwè varyasyon ki egziste ant chat la ak chat lan se yon varyasyon lib. Men Valdman limenm (1991) pa $\mathrm{t}$ sèlman rete la, nan yon etid pilòt li fè, li montre varyasyon sa a se yon chanjman $\mathrm{k}$ ap fèt nan lang nan, e gwoup moun ki ta alatèt chanjman sa a se ta jenn moun ki bileng yo. Nou te reprann menm etid sa a ankò nan lane 2014 sou yon echèl ki pi djanm epi ki pi byen kanpe, kote tout gwoup sosyal yo te byen reprezante (egzanp: iben /riral, mono-/bileng). Nan fouye chache, nou te rive remake ke chanjman sa a ta ta chita sou yon modèl ki byen dekri nan nosyon analoji nou jwen nan deskripsyon Sosi (Saussure) (1916) a, sa vle di nazalizayon detèminan sa yo rive posib apati resanblans ak asosyasyon lokitè yo fè ak gwoup nominal tankou jenou an ak pitimi an. Sepandan, malgre fenomèn nazalizasyon detèminan sa a ta sanble fin gaye nan prèske tout kontèks lengwistik yo (egz. diri an, pè an, mizik lan), nou kapab remake geynyen yon rezistans toujou onivo vwayèl ki ba yo (egz. papa a / *papa an oswa anana a /*anana an). Rezon ki eksplike absans 
nasalizasyon nan deteminan an apre mo sa yo se akòz sa nou ta rele ekonomi atikilatwa.

\section{Abstract}

In this study I investigate the role of analogy in the sociolinguistic and cognitive aspects of a morphophonological change in progress in Haitian Creole, precisely the nasalization of the postposed determiner. Dejean (1980) and Joseph (1984) associated the alternation between chat la and chat lan 'the cat' with a free variation. Valdman (1991) later conducted a pilot study which indicated that this variation was a change in progress being led by young urban bilingual speakers. Again, we took up the same study in 2014 on a larger scale with more rigor, where all classes of subjects (urban / rural, mono- / bilingual) were included. We noted that this change was based on a model duly described by the Saussurean's notion of analogy (1916); that is, by association and resemblance to noun phrases such as jenou an 'the knee' and pitimi an 'the millet'. However, even though the nasalization of the determiner has become widespread in almost every linguistic context (ex. diri an 'the rice', pè an 'the priest', mizik lan 'the music'), there is still some resistance in the environment of the low vowels (ex. papa $a / * a n$ 'the father', anana $a / * a n$ 'the pineapple'). This resistance persists because of articulatory economy.

\section{Introduction}

Cette étude s'inscrit dans le cadre d'une recherche de terrain qu'Albert Valdman (1991) a menée en Haïti, recherche par laquelle il a constaté que plusieurs locuteurs haïtiens avaient tendance à nasaliser le déterminant la en contexte non nasal. Ce phénomène s'observe lorsqu'un locuteur nasalise le déterminant à la suite d'un mot se terminant par un phonème oral (ex. tab la devient tab lan « la table », et diri a se prononce diri an « le riz »). Se basant sur une approche sociolinguistique, Valdman (1991) a prédit que ce phénomène constituerait un changement linguistique en cours, et indiqué que les jeunes bilingues de Port-au-Prince en seraient les principaux initiateurs. Ce lien qu'a établi Valdman entre la nasalisation en contexte non nasal et les facteurs sociaux (ex. âge, éducation, etc.) a marqué une nouveauté dans le domaine sociolinguistique en créole haïtien $(\mathrm{CH})$.

Au cours d'une nouvelle étude de terrain menée en Haïti, en 2014, nous avons observé ce même phénomène de généralisation de la nasalisation en contexte non nasal. Toutefois, en plus du lien qui existait entre le taux de nasalisation et la tranche d'âge du locuteur, il a été aussi remarqué que ce phénomène était fortement conditionné par des contextes linguistiques, résultant plus précisément de la hauteur de la voyelle que comprend la syllabe précédant le déterminant. Nous avons, par exemple, remarqué qu'avec les syllabes ouvertes les cas de nasalisation se produisent le plus souvent au contact des voyelles de $1^{\text {er }}$ degré d'aperture (ex. $/ \mathrm{i} /$ et $\left./ \mathrm{u} /\right)^{1}$ comme dans les mots : diri « riz », lakou 'la cour', et presque jamais dans le cas des mots terminés par la voyelle /a/ dans un mot comme : papa 'père'. Mais lorsque ces voyelles sont suivies d'une consonne finale, la nasalisation du déterminant devient possible avec toutes les voyelles, y compris [a] (ex. patat «patate »). L'une des principales découvertes faites dans cette étude révèle que ce changement linguistique en cours observé par Valdman (1991) s'étend non seulement chez les citadins bilingues vivant dans les régions

\footnotetext{
${ }^{1}$ Voyelles fermées ou hautes $\left(1^{\text {er }}\right.$ degré d'aperture: [i], [u])

Voyelles mi- fermées ( $2^{\text {ème }}$ degré d'aperture : [e], [o]

Voyelles moyennes ( $3^{\text {ème }}$ degré d'aperture : $[\varepsilon][0)$

Voyelle ouverte or basse (4ème degré d'aperture: [a])
} 
métropolitaines mais aussi chez les unilingues résidant dans les zones rurales du pays.

Dans cette perspective, nous soutenons que ce changement linguistique ne constitue pas une violation des règles générales de la langue, qui, d'après Saussure (1916), suppose «un modèle et son imitation régulière. Une forme analogique est une forme faite à l'image d'une ou plusieurs autres d'après une règle déterminée ». Nous partons de ce principe saussurien pour démontrer que la nasalisation du déterminant en contexte non nasal suit une régularité qui a été préalablement établie dans la langue, notamment dans la détermination des lexèmes en contexte nasal. Le modèle exemplaire de ce changement se trouve dans la distribution même du déterminant la en $\mathrm{CH}$. Par exemple, dans les syllabes vocaliques de structure $\mathrm{C}^{\mathrm{N}} \mathrm{V}$ (consonne nasale + voyelle), l'occurrence des formes nasales du déterminant se fait de manière catégorique lorsque les voyelles de $1^{\mathrm{er}}$ degré d'aperture sont en contexte de nasale précédente (ex. pitimi an « le millet», jenou an « le genou»), mais la nasalisation ne se produit pas lorsque c'est la voyelle de $4{ }^{\text {ème }}$ degré d'aperture qui se trouve au même contexte (ex. anana $a$ «l'ananas »). Et ceci se fait de manière systématique. Nous constatons également la variation entre l'oral et le nasal après les syllabes de type $\mathrm{C}^{\mathrm{N}} \mathrm{VC}$ (consonne nasale + voyelle orale + consonne orale) comme dans klinik la/lan, «le clinique », almanak la/lan « le calendrier ». Donc, nous démontrons que la nasalisation du déterminant en contexte non nasal suit le modèle nasal décrit précédemment, c'est-à-dire qu'elle apparaît avec toutes les voyelles du $\mathrm{CH}$ en syllabe fermée (i.e. CVC), mais en syllabe ouverte de type CV, il y a une contrainte vocalique sur les niveaux de degré d'aperture interdisant son occurrence avec la voyelle [a], et qui est favorable aux voyelles ayant pour trait [+haut], particulièrement $[\mathrm{i}]$ et $[\mathrm{u}]^{2}$. Même si des formes nasales et orales varient après un phonème oral, lorsqu'elles sont influencées par des facteurs sociaux, stylistiques et idiosyncratiques, il semble que le locuteur natif haïtien n'est pas libre de dire ce qu'il veut où il veut, car la langue lui impose de manière générale des contraintes linguistiques (Prince \& Smolensky 1993) dont les différents ordonnancements sont bien évidemment possibles dans certains contextes.

\section{Analogie linguistique}

En linguistique, l'analogie se définit comme un processus par lequel une forme devient de plus en plus semblable à une autre par laquelle elle a une certaine association (Arlotto 1972). Les études psycholinguistiques du phénomène tiennent surtout comptent de l'aspect cognitif généralement connu sous le nom de «mapping » qui, selon Ripoll et Coulon (2001 : 290) est lié à la capacité générale d'identifier des similitudes profondes non triviales et non apparentes en surface. Gentner (1989) et Jeziorski (1993) suggèrent que c'est grâce à un usage systématique du raisonnement par analogie, que la mise en relation d'un cas connu (la source) et d'un cas inconnu (la cible), la recherche de similitudes est possible. Parmi les cas d'analogie observés on recense au moins deux types: l'analogie proportionnelle et l'analogie de nivellement.

\subsection{L'équation de la quatrième proportionnelle}

Une forme $\mathrm{A}$ ressemble à une forme $\mathrm{B}$ comme $\mathrm{C}$ ressemble à $\mathrm{D}$, autrement dit, $\mathrm{A}$ est à $\mathrm{B}$ ce que $\mathrm{C}$ est à $\mathrm{D}$. L'équation de la quatrième proportionnelle est le principe par lequel l'on forme des mots, elle se caractérise par cette relation de deux à deux. Par exemple, en anglais le verbe drive 'conduire'

\footnotetext{
${ }^{2}$ La nasalisation du déterminant en contexte non nasal est aussi possible avec les voyelles de $2^{\mathrm{e}}$ et $3^{\text {e }}$ dégrée d'aperture ; cependant d'après nos données statistiques, les locuteurs montrent une plus forte préférence pour les voyelles de $1^{\mathrm{e}}$ degré d'aperture (Tézil, à paraître).
} 
(dont le temps du passé est drove) ressemble au verbe dive 'plonger' (avec dived au passé). Par analogie au premier verbe, le second verbe a tendance à adopter la forme irrégulière (i.e. dove au lieu de dived) (Campbell 1998).

\subsection{L'analogie de nivellement}

Le deuxième type d'analogie se reconnait sous divers termes tels que la création analogique ou le nivellement analogique en fonction de ce qui est en jeu (Trask 1996 :106) - 109). Le rôle de l'analogie de nivellement est de rendre un système quelconque moins irrégulier soit pour des raisons d'économie articulatoire ou des raisons d'économie de la mémoire.

Au cours du développement du latin parlé le [a] accentué du verbe amare 'aimer' en syllabe ouverte était transformé en une diphtongue [ai] en ancien francais, tandis que le [a] inaccentué n'avait pas été affecté (ex. amons, amez).

Plus tard dans le français contemporain, nous voyons qu'à partir d'un procédé analogique, la forme accentuée va régulariser tout le paradigme. C'est donc l'effet analogique qui nivèle le paradigme. Soit le tableau suivant :

\begin{tabular}{|l|c|c|c|}
\hline & Latin parlé & Ancien français & Français contemporain \\
\hline $1 \mathrm{sg}$ & ámo & aim & Aime \\
\hline $2 \mathrm{sg}$ & ámas & aimes & Aimes \\
\hline $3 \mathrm{sg}$ & ámat & aimet & Aime \\
\hline $1 \mathrm{pl}$ & amámus & amons & Aimons \\
\hline $2 \mathrm{pl}$ & amátis & amez & Aimez \\
\hline $3 \mathrm{pl}$ & ámant & aiment & Aiment \\
\hline
\end{tabular}

Tableau 1. - Lenivellement analogique en français, adaptation et traduction du tableau de Trask (2007: 138)

En anglais, également, auparavant le mot sword 'épée' se prononçait avec un [w], un peu plus tard le [w] a disparu lorsqu'il se trouvait entre [s] et une voyelle postérieure comme swore (passé simple du mot verbe swear 'jurer'). Le [w] a été rétabli et réintroduit dans le mot swore par nivellement analogique, c'est-à-dire par association au temps présent swear dont la forme retenait encore le [w], ce qui pousse McMahon (1994 : 74) à suggérer qu'au cours du processus de changement sonore, l'analogie pourrait s'interférer au niveau de l'output (i.e. en surface), même si elle ne l'inverse pas entièrement.

\subsection{Comment distinguer le changement par analogie des autres développements phonologiques?}

Généralement les linguistes reconnaissent deux types majeurs de développement phonologique: le changement à conditionnement strictement phonétique (i.e. les changements au niveau des sons tout court, du point de vue néogrammairien), qui est régulier, et le changement par analogie, qui est irrégulier (Hualde 2006, Campbell 1998). Alors pour déterminer si un changement donné est fondé sur le contexte phonétique ou analogique, Hualde (2006: 451) a proposé de prendre en compte quatre critères de classification, notamment les deux premiers qui suivent :

1. Les changements analogiques qui, de par leur nature même, sont clairement non phonétiques.

2. Les changements analogiques qui ne sont pas manifestement différents dans leurs effets de changements sonores déclenchés phonétiquement. 
Les cas de changement strictement conditionnés par la phonétique sont provoqués le plus souvent en raison des faits articulatoires. Hualde (2006) a remarqué que la palatalisation de $/ \mathrm{n} /$ et de $/ 1 /$ et celle d'autres consonnes coronales après /i/ retrouvées dans de nombreux dialectes basques, comme dans mina > miña 'la douleur', mutila > mutilla constituent des changements sonores qui sont phonétiquement conditionnés. De même, en $\mathrm{CH}$, la voyelle /a/ de la première syllabe du mot kana 'canard' peut être nasalisée par une règle d'assimilation régressive et devient kanna. Certains créolistes (Cadely 2002, Valdman \& Iskrova 2003) ont aussi proposé la présence d'une règle d'assimilation progressive dans le processus de nasalisation du déterminant en CH (par exemple : dra a 'le drap' vs dan an 'la dent').

Les changements analogiques, cependant, résultent généralement d'autres processus (comme la réanalyse et le nivellement) qui ne sont pas forcément phonétiquement conditionnées.

Hualde (2006) a également remarqué que la consonne initiale /t/ du mot basque tarte, dont l'origine vient du mot arte 'entre', aurait été le produit de la réanalyse d'une consonne provenant de la racine d'un mot composé betarte « espace entre les yeux » et qui s'est répandue en une consonne épenthétique dans d'autres formes composées tels que bi-t-arte «entre deux », et finalement s'est réanalysée en un morphème initial.

Les changements analogiques peuvent être aussi provoqués par le processus de nivellement. Comme nous l'avons vu précédemment (tableau supra), il s'agit d'un processus où les termes d'une série se conforment à un modèle dominant dont l'un des objectifs majeurs est la simplification du système. Wetzels (1981 : 38) note que la perte d'une règle morpho-phonologique par exemple représente un cas de simplification allant de pair avec la lexicalisation de la forme qu'elle a produite. Toutefois, comme l'a fait remarquer Albright (2008: 150), ce ne sont pas tous les cas de changements linguistiques qui peuvent être directement liés à la simplification :

The idea that analogy always results in grammar simplification is tantalizing, but unfortunately, there are many changes that cannot be straightforwardly analyzed as simplification.

Albright (2008) suppose par exemple que le changement qui s'opère dans la première personne du singulier du paradigme du verbe allemand gebe « donner» (gibe gebe) (quoique considéré comme un procédé analogique) n'exclut pas la possibilité d'une analyse alternative basée sur une règle d'alternation où $e \sim i$, ou une explication basée sur le conditionnement du contexte phonétique. 


\section{(a) Contamination}

L'usage du terme contamination ne fait pas référence à la fréquence d'emploi d'un mot ou d'une forme mais plutôt aux cas dans lesquels un mot ou un morphème est modifié ou créé par correspondance avec un autre mot avec lequel il coexiste (Hualde 2006, Trask 1996). Un autre terme qui est parfois utilisé pour décrire le processus de contamination est l'analogie sporadique. Il a été montré par exemple que la consonne inter-dentale voisée de l'anglais / $/$ / (comme dans le mot father [faðə(r)] «père ») est un cas de contamination puisque d'après la Loi de Grimm et de Verner, au cours du changement sonore la consonne intervocalique [t] du mot original *poter devrait d'abord se voiser en [d] avant d'aboutir à une consonne fricative [ð] (Hogg 1979, MacMahon 1994). Toutefois, il n'existe aucune preuve de l'existence d'une telle consonne dans l'anglais moderne (*[fadə(r)]). MacMahon (1994 : 74-75) pense donc que la présence de [ð] dans le mot father est le résultat d'un changement par analogie avec le mot brother «frère ». Il a par ailleurs remarqué que cet exemple de contamination analogique se réalise particulièrement dans les mots qui ont un lien sémantique (ex. parenté), ainsi que dans ceux faisant partie d'une liste commune tels que les jours de la semaine, les mois de l'année, etc. (Anttila 1977). Au regard de ce qui vient d'être exposé, l'on dira en résumé, que la contamination analogique est un changement qui ne suit pas forcément les règles phonologiques.

\section{(b) Hypercorrection}

L'hypercorrection est parfois perçue comme une erreur langagière. Toutefois, il existe des cas d'hypercorrection où le motif est stylistiquement, socialement et même contextuellement (i.e. contextes formel et informel) conditionné. Quoique l'usage d'une forme hypercorrigée soit un processus qui peut se faire consciemment ou inconsciemment, on observe qu'en général la sélection de cette forme se dirige vers la forme perçue comme étant la plus prestigieuse, comme le décrit Trask (1996 : 112) :

The other special type of analogy is hypercorrection. This occurs when a speaker deliberately tries to adjust his or her own speech in the direction of another variety perceived as more prestigious but 'overshoots the mark' by applying an adjustment too broadly.

En anglais, par exemple, on a souvent constaté une tendance chez certains locuteurs à alterner le pronom sujet de la première personne du singulier $I$ 'je' avec le pronom complément me 'moi' dans des contextes où la langue généralement exige la forme ' $m e$ ' en position de complément (ex. phrase grammaticale : He called John and me. vs phrase hypercorrigée : He called John and I. 'Il a appelé Jean et moi'). De même, dans le cas du CH, les voyelles antérieures arrondies du français de référence (ex. [œ], [ø], [y]) varient avec les voyelles antérieures non arrondies (ex. [e], $[\varepsilon],[i])$ chez beaucoup de locuteurs bilingues haïtiens (ex. duri / diri, bèu / bè, deu / de) (Schieffelin \& Doucet 1994, Valdman 2015). Certains locuteurs haïtiens considérés comme moins scolarisés et unilingues ont aussi adopté ces voyelles arrondies. Cependant, il est souvent arrivé qu'ils les généralisent là où la voyelle non arrondie est obligatoire (ex. yon kalite bleu) au lieu de yon kalite ble ou encore Nou seure li au lieu de Nou sere li.

\section{L'analogie et les changements linguistiques: quelques repères théoriques}

Toutes langues subissent des changements au fil du temps. Le français, l'espagnol, l'anglais, etc., ont tous connu des changements continus tout au long de leur existence. En termes de prononciation, par exemple, la voyelle 
[a:] du mot vieil anglais [ha:m] 'domicile' s'est changé en [ho:m] en moyen anglais, puis [howm] en anglais moderne. Dans les études diachroniques, il a aussi été observé que les changements affectant la voyelle [a:] sont produits non seulement dans le mot [ha:m] mais aussi dans d'autres mots comme boat 'bateau', oath 'serment' et 'stone' pierre' (O'Grady et al. 2005 : 246).

\begin{tabular}{|l|l|l|l|}
\hline Ancien anglais & Moyen anglais & Anglais contemporain & \\
\hline$[$ ba:t $]$ & {$[$ bo:t } & {$[$ bowt $]$} & 'boat' \\
{$[\mathrm{a}: \theta]$} & {$[\mathrm{s} \theta]$} & {$[$ ow $\theta]$} & 'oath' \\
[sta:n] & {$[$ sto:n] } & {$[$ stown $]$} & 'stone' \\
\hline
\end{tabular}

Tableau 2.- Changements affectant le phonème [a:] de l'ancien anglais (O'Grady et al. 2005 : 247)

La manière dont le changement s'est régularisé et systématisé est présenté tableau 2, suivant la même règle du modèle (i.e. le mot home). Les facteurs cognitifs jouent un rôle essentiel dans le changement de toutes les composantes de la grammaire de la langue. Sur cette base, l'analogie constitue l'une des sources de ce changement. D'après certains linguistes (O'Grady et al. 2005), elle reflète la préférence des locuteurs pour des formes régulières plutôt que celles qui sont irrégulières. Elle implique par-là l'extension ou la généralisation d'une régularité sur la base de l'inférence que si les éléments sont similaires dans certains aspects, ils doivent être aussi bien semblables dans d'autres. (O'Grady et al. 2005, Trask 2007).

L'analogie constitue un phénomène assez répandu jouant un rôle important dans l'acquisition et le développement des régularités (surtout chez l'enfant). Très souvent, les locuteurs créent des formes en appliquant une analogie avec un nombre beaucoup plus restreint des formes existantes. Quoique restreintes, ces formes sont puissantes, en raison de leur fréquence, leur productibilité ainsi que leur prédictibilité. Bybee (2008: 108) a remarqué que les changements qui se produisent dans les contextes phonétiques affectent les mots qui ont une fréquence élevée avant ceux qui ont une fréquence basse.

Les changements linguistiques (particulièrement les changements au niveau des sons du langage) se produisent pour des raisons de convenance ou d'aisance articulatoire aussi connu sous le nom d'économie articulatoire (Haspelmath 2008 : 214). Les néogrammairiens par exemple ont noté que tous les changements de sons réguliers constituent des faits qui sont motivés mécaniquement et physiologiquement (McMahon 1994). Toutefois, l'interaction entre le changement de ces sons et l'analogie est mieux encapsulée dans le paradoxe du linguiste américain Edgar H. Sturtevant (1947:109) qui a affirmé que le changement sonore est régulier mais crée une irrégularité, alors que l'analogie est irrégulière mais crée de la régularité. Cette étude porte principalement sur les changements synchroniques, la nature des modèles linguistiques dans une langue particulière à un moment donné. Mais pour mieux comprendre les modèles synchroniques, nous pensons qu'il serait parfois utile de considérer l'aspect diachronique des changements analogiques (Haspelmath 2010). Et quoique l'analogie se manifeste à la fois dans les changements diachroniques et synchroniques, l'attestation de ces changements linguistiques est plus pertinente sur le plan diachronique en raison du fait qu'il est difficile (sur le plan synchronique) de s'assurer que le changement d'une forme aboutira à la régularisation et au changement d'autres formes. En général les changements linguistiques se développent au cours d'une longue période de temps puisque dans beaucoup de cas c'est le locuteur qui en décide, même inconsciemment. Toutefois, que ce soit du point de vue synchronique ou diachronique, les changements 
analogiques sont motivés par le désir du locuteur de s'exprimer économiquement. Haspelmath (2008 : 214) perçoit ce désir comme des contraintes sur tout comportement rationnel constraint on any rational behavior et qui s'étend au-delà du temps et des générations.

\section{Le déterminant en $\mathrm{CH}$ : variation morphémique et distri- bution}

Les linguistes sont unanimes à reconnaitre que dans les créoles français de la Caraïbe, la forme sous-jacente /la/ du déterminant s'est diachroniquement développée en divers allomorphes (oral et nasal) observés particulièrement dans les variétés du sud de la Dominique, en Haïti, en Martinique, à SainteLucie et à la Trinidad (Taylor 1968, Dejean 1980, Damoiseau 1984). Bernabé (1983) a cependant remarqué que la ne varie pas en Guyane, en Guadeloupe et dans la partie nord de la Dominique. Dans le tableau cidessous ne sont présentés que les allomorphes en contexte oral et non ceux apparaissant en contexte nasal, qui sont d'ailleurs étudiés dans des paragraphes ci-dessous.

\begin{tabular}{|l|c|c|c|}
\hline & Guyane & $\begin{array}{c}\text { Haïti ; sud de la } \\
\text { Dominique, Mar- } \\
\text { tinique, Sainte- } \\
\text { Lucie, Trinidad }\end{array}$ & $\begin{array}{c}\text { Guadeloupe, } \\
\text { nord de la } \\
\text { Dominique }\end{array}$ \\
\hline Après voyelle orale & {$[\mathrm{a}]$} & {$[\mathrm{a}]$} & {$[\mathrm{la}]$} \\
\hline Après consonne orale & {$[\mathrm{a}]$} & {$[\mathrm{la}]$} & {$[\mathrm{la}]$} \\
\hline
\end{tabular}

Tableau 3.- Le déterminant la dans les îles créolophones (Extrait de Bernabé 1983)

Dans les créoles à variation morphémique tels que le $\mathrm{CH}$, celui du sud de la Dominique, la Martinique, de Ste-Lucie, et de Trinidad, le choix des morphèmes est influencé par le phonème du mot précèdent. Après une voyelle orale, le déterminant se réalise en [a] et en [la] après une consonne orale. 


\section{Distribution de la en $\mathbf{C H}$}

Parmi les régions créolophones de la Caraïbe, Haïti est l'un des pays où le déterminant la contient des variations allomorphiques les plus étendues : [1a], [a], [lã], [ã], [nã] (Sylvain 1936, Faine 1937, Hall 1953, Valdman 1978, Dejean 1980, Joseph 1984, Jean-Baptiste 1992, Cadely 1996, DeGraff 2007). Soient les exemples suivants illustrant l'apparition de ces morphèmes ${ }^{3}$.
(a) /tab/ 'table'/tab la/ [tab la] 'la table'
(b) /bagaj/ 'truc' /bagaj la/bagaj la] 'le truc'
(c) /papa/ 'père' /papa la/ [papa a] 'le père'
(d) /diri/ 'riz'/diri la/ [diri a] 'le riz
(e) /bã/ 'banc' /bã la/ [bã ã] 'le banc'
(f) $/ \mathrm{ma} / \mathrm{in} /$ 'voiture' /ma $\mathrm{jin} /[\mathrm{ma} / \mathrm{in}(\mathrm{n} / \mathrm{l}) \tilde{a}]$ 'la voiture'

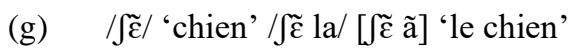
(h) /bãk/ 'banque' /bãk la/ [bãk lã/la] 'la banque'
(i) $/$ mõt/ 'montre' /mõt la/ [mõt lã/la] 'la montre'
(j) /lam/ 'arbre à pain' /lam la/ [lam (n/l)ã] 'l'arbre à pain'
(k) /zenu/ 'genou'/zenu la/ [zenu ã] 'le genou'
(l) /lame/ 'armée' /lame la/ [lame a] 'l'armée'

Tableau 4.- Distribution du déterminant la en $\mathrm{CH}$

Dans les exemples (4) et (4b), l'allomorphe [la] fait son apparition après les mots se terminant par une consonne orale ou une semi-consonne (ex. tab la 'la table', bagay la 'la chose'), alors qu'en (4c-d) c'est l'allomorphe [a] qui se produit après une voyelle orale (ex. papa $a$ 'le père'). Dans les cas de nasalisation, la voyelle nasale [ã] suit les mots qui se terminent par une voyelle nasale (ex. ban an 'le banc'), tandis que le morphème nasal [nã] apparaît après une consonne nasale (ex. machin nan 'la voiture) A noter qu'il existe parfois une variation linguistique entre les allomorphes [lã] et [la], après les mots contenant une voyelle nasale suivie d'une consonne orale, comme indiqué dans $4 \mathrm{~g}$ et $4 \mathrm{~h}$ (ex. mõt lã lla 'la montre'). Il existe encore une variation entre les allomorphes [nã] et [lã] dans les mots ayant une consonne nasale en position finale comme dans : machin nã /la 'la voiture' (Dejean 1980 : 143). Nous constatons dans l'exemple (4k), que c'est la forme nasale [ã]qui suit le mot jenou [zenu] 'genou', et non la voyelle orale [a]. Cette observation a été faite par Dejean (1980) et Valdman (1991) qui supposent que la voyelle serait catégoriquement nasalisée après les mots dont la syllabe finale comprend une consonne nasale suivie d'une voyelle haute comme /i/ et $/ \mathrm{u} /$.

Lorsque cette voyelle a une hauteur moyenne ou basse comme [e] et [a] c'est le morphème vocalique oral [a] qui apparaît (ex. lame 'l'armée', anana a 'l'ananas), quoique la voyelle soit précédée d'une consonne nasale. Ceci sous-entend qu'il existe un lien entre la nasalisation et la hauteur de la voyelle en $\mathrm{CH}$. Si nous admettons par exemple que les déterminants placés après les mots à voyelles hautes comme jenou 'genou' et mi 'mur' subissent eux-mêmes une nasalisation par assimilation sous l'influence de la consonne nasale, nous sommes persuadés que l'absence de ce phénomène (à savoir, la nasalisation des déterminants placés après des mots ayant des voyelles basses

\footnotetext{
${ }^{3}$ Les exemples sont présentés en phonologie (entre barres obliques) et en phonétique (entre crochets).
} 
tels que anana a et non anana an 'l'ananas') crée une autre réalité dans la langue.

\section{Variation entre les formes orale et nasale du determinant en contexte non nasal}

En plus de la description générale du déterminant défini présentée au tableau 2 , des cas récurrents de nasalisation ont été répertoriés dans des contextes non nasal. Par exemple, les allomorphes nasalisées an [ã] et lan [lã] ([1 (ã)]) alternent avec leurs équivalents oraux [a] et [la] (dorénavant [1 (a)]) après les mots terminant par un phonème oral. Il semble, dans ce cas, que les locuteurs haïtiens procèdent par analogie avec le modèle de nasalisation traditionnellement admis en contexte nasal : machin lan $\sim$ la machine, melon an le melon, pour nasaliser même des mots placés en contexte non nasal. On recense facilement des cas de variation comme : rezo an / rezo a, chodyè an / chodyè $a$, jwèt lan / jwèt la..., au lieu de rezo $a$, chodyè a et jwèt la, uniquement. Cela semble provenir de la capacité des locuteurs à créer de nouvelles formes et à innover ou généraliser les structures déjà établies dans la langue. Nous reviendrons plus loin sur cette question pour approfondissement.

L'observation énoncée plus haut a été rapportée dans plusieurs études (Sylvain 1936, Faine 1937), ainsi que dans la thèse de Dejean (1980 : 143). En voici deux exemples :

Variation entre les formes orale et nasale du déterminant après un segment oral
(1a) tèt la /lan 'la table'
(1b) pitia/an 'le petit'

L'usage des formes nasales du déterminant en contexte non nasal (i.e. après des segments oraux) est un phénomène qui a été aussi attesté dans plusieurs études antérieures (Sylvain 1936, Faine 1937, Hall 1953, Valdman 1978, Dejean 1980, Jean-Baptiste 1992, Cadely 1996, Nikiema 1999 et DeGraff 2007). Cependant, à notre connaissance, seules les études de Dejean (1980), de Joseph (1984) et de Valdman (1991) l'ont explicitement lié à une tendance linguistique. Selon Dejean (1980), l'emploi de morphèmes oraux et nasals constitue une variation libre, alors que Joseph (1984: 87) l'a un peu plus tard caractérisé de variation stylistique observée chez des locuteurs haïtiens éduqués dans le but de s'approprier un discours «plus ou moins recherché ». L'affirmation de Dejean (1980) et celle de Joseph (1984) ont fait avancer les débats, mais elles n'ont pas néanmoins été étayées par des données empiriques. Il a fallu attendre l'étude sociolinguistique de Valdman (1991) pour confirmer la montée de ce phénomène. Se basant sur des données empiriques, Valdman a pu démontrer un lien entre le taux de nasalisation de la en contexte non nasal et la tranche d'âge des locuteurs. 
La méthodologie de Valdman se base sur une approche variationniste selon laquelle les locuteurs sont classifiés selon l'âge, le sexe et l'éducation. Son étude comprenait huit hommes et huit femmes. Tous les locuteurs participant à l'étude étaient bilingues (créole et français) issus de la classe moyenne, universitaires et résidents de Port-au-Prince. En ce qui concerne l'âge, la moitié des sujets se situaient entre 18 et 25 ans (juniors) et l'autre moitié entre 40 et 60 ans (seniors). Les données contiennent une heure de conversation lancée par les 10 minutes d'intervention dirigée avec chaque locuteur. Voici un exemple illustrant la variation des formes orales et nasales (Valdman $1991: 84)$ :

(2) Ou pa ka konte sou sinema-a pou di ke lavi-an bèl

2sg. Neg. pouvoir compter Prep cinema-DET Prep dire Comp vie-DET belle «On ne peut pas compter sur le cinéma pour se dire que la vie est belle.»

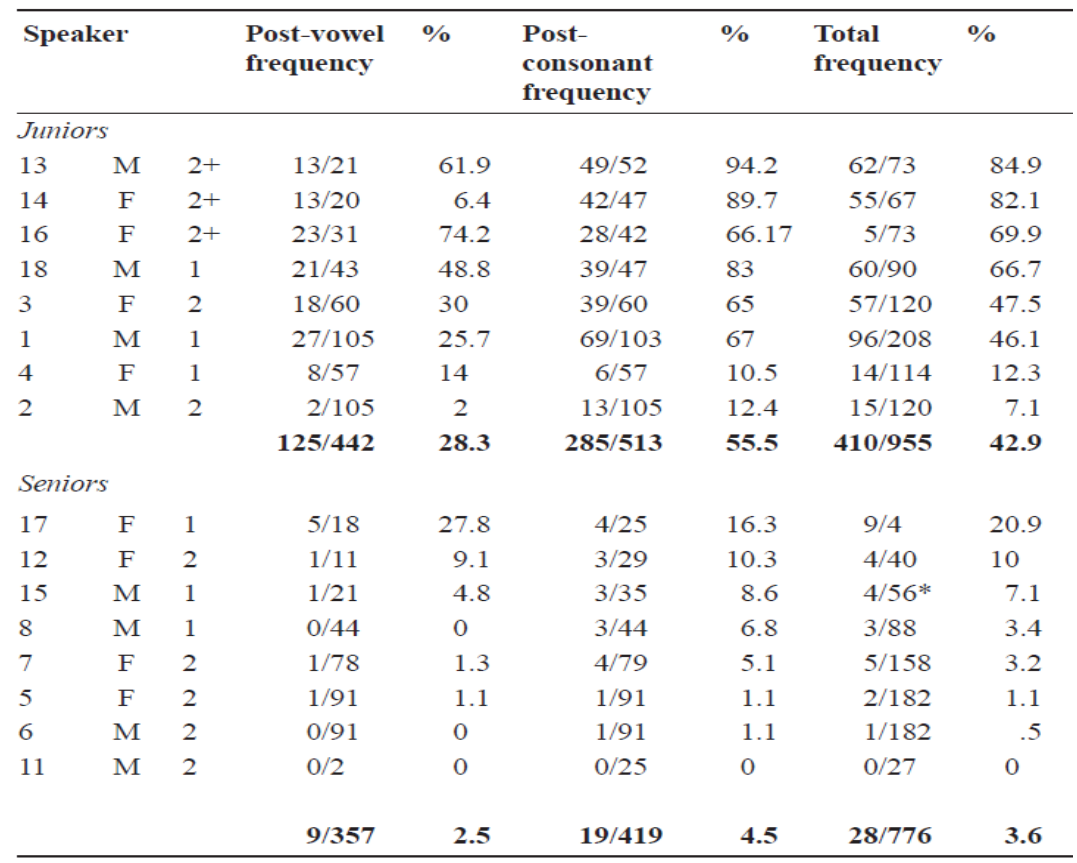

Tableau 54 .- Nasalisation du déterminant défini chez les locuteurs résidant à Port-au-Prince (Valdman 1991 : 84 et 2015 : 332)

En (2), le déterminant est produit à deux reprises dans le même énoncé. La même forme varie dans le même contexte, autrement dit, le morphème du déterminant défini est oral après sinema 'cinéma' mais nasal après lavi 'la vie'. A l'aide des données quantitatives recueillies, Valdman (Tableau 5 cidessus) a pu trouver un lien entre l'âge du locuteur et l'extension de la nasalisation aux segments oraux. Il a constaté que les morphèmes nasals lan [lã] et an [ã] apparaissent en contexte non nasal plus fréquemment chez les locuteurs les moins âgés que les plus âgés. Valdman (1991: 84) montre une différence significative entre le pourcentage de nasalisation chez les sujets plus jeunes $(42,9 \%)$ et celui des sujets plus âgés $(3,6 \%)$.

Valdman (ibid.) fait deux remarques en conclusion :

1. puisque le pourcentage de nasalisation plus élevé chez les jeunes locuteurs, il y aurait un changement linguistique en cours, dont les plus jeunes seraient le chef de file,

2. le changement affecte les consonnes plus rapidement que les voyelles, puisqu'il y avait plus d'occurrences de nasalisation dans des mots terminant par une consonne. 


\section{La nasalisation des déterminants en contexte non nasal: un changement linguistique}

Outre les études de Valdman (1991), des données encore plus récentes que nous avons recueillies en 2014 nous ont permis non seulement de confirmer la généralisation de la nasalisation en contexte non nasal du determinant chez les jeunes locuteurs mais aussi de determiner les facteurs linguistiques qui favorisent ce changement. Notre étude pilote (Tézil inédit) a aussi adopté une approche empirique afin de déterminer si la nasalisation des déterminants en contexte non nasal est en réalité influencée par des facteurs sociolinguistiques. Dans cette étude pilote, nous avons abordé deux questions majeures :

1. les facteurs linguistiques - la nasalisation des déterminants est-elle plus favorisée dans certains environnements linguistiques que dans d'autres?

2. les facteurs sociaux - Le changement en cours proposé par Valdman (1991) s'est-il étendu au-delà des locuteurs bilingues urbains aux locuteurs unilingues ruraux?

\section{Méthodologie}

\subsection{Comment déterminer les contextes nasals et non nasals?}

Dans la description générale du déterminant du $\mathrm{CH}$, les contextes nasals et non nasals sont déterminés à partir du dernier phonème du mot précédant le déterminant. Par exemple, après les phonèmes [n] et $[\mathrm{m}]$, c'est le morphème nan [nã] ou lan [lã] qui apparaît (machin nan 'la voiture'). Cette description n'indique pas cependant qu'il y a des cas de nasalisation dont le contexte va au-delà du dernier phonème. Par exemple, le mot bank 'banque' peut être suivi soit des allomorphes oral [la] ou nasal [lã]. Nous supposons qu'il y a deux contextes possibles. Le locuteur qui ne nasalise pas le déterminant prend en compte le phonème oral $[\mathrm{k}]$ tandis que celui qui nasalise le déterminant prend en compte non seulement le phonème [k] mais aussi la voyelle nasale [ã].

Un autre argument probant à considérer est le choix du morphème [ã] après zanmi et [a] après diri. Si le choix du déterminant n'est déterminé qu'en fonction du dernier phonème [i], pourquoi existe-t-il cette variation entre les deux formes du déterminant ? Et même au cas où /i/ serait nasalisée en [ĩ] dans le mot zanmi, du point de vue phonologique elle n'est pas différente du phonème /i/ que nous retrouvons dans le mot diri, puisque le trait nasal peut être transmis par assimilation à la consonne nasale $[\mathrm{m}]{ }^{4}$. Cette vue a été en effet adoptée dans notre étude puisqu'il n'existe jusqu'à présent aucune preuve convaincante qui confirme le statut phonémique de $\tilde{\imath}$ et $\tilde{u}$ dans les mots pitimi et jenou (Dejean 1980, Valdman 1991, Cadely 1994).

C'est sur cette base que notre méthodologie établit les contextes nasals et non nasals du point de vue de la syllabe finale du mot précédant le déterminant (voir tableau 6), une approche différente de celle du tableau 4. Ceci nous permettra par exemple de mieux saisir le lien entre le phonème final et sa relation avec les autres composants de la syllabe. L'une des questions qui nous intéresse c'est aussi de déterminer la différence

4. Le statut des voyelles nasales hautes est un phénomène qui continue de faire l'objet de débat entre certains linguistes particulièrement Tinelli (1981), Dejean (1980), Valdman (1991) et Cadely (1994). Dans le cas du mot pitimi, Tinelli (1981) suggère que /i/ aurait déjà été nasalisé par assimilation progressive avec la consonne nasale [m], ce qui crée alors un contexte nasalisé pour que le déterminant lui-même soit nasalisé aussi. Comme le souligne Valdman (communication personnelle), Tinelli (1981) soulève une question très pertinente car il existe des preuves de nasal /î/ et /ũ/ (par exemple, oungan 'prêtre du vaudou'). Cependant, Dejean (1980) a aussi observé qu'il n'existe pas de contraste phonologique entre les paires minimales [bũda] /[buda] et même [fumi] / [fũmi]. En d'autres termes, le sens ne change pas. Par conséquent, l'argument sur le statut phonémique des voyelles nasales /i/ et /u/ est difficile à soutenir. 
d'interaction lorsque la consonne de l'attaque est nasale par rapport à la consonne orale (C). Par exemple, lorsque le /i/ final dans les mots diri et zanmi attire le déterminant [ã], nous nous demandons si c'est au même niveau, et qu'est-ce que cela nous signalerait sur les plans phonétique et phonologique.

\begin{tabular}{|c|c|c|c|c|c|}
\hline $\begin{array}{l}\text { Contextes } \\
\text { nasals }\end{array}$ & Syllabes & Exemples & $\begin{array}{l}\text { Contextes non } \\
\text { nasals }\end{array}$ & Syllabes & Exemples \\
\hline $\begin{array}{l}\text { Consonne } \\
\text { orale+ voyelle } \\
\text { nasale }\end{array}$ & $\mathrm{CV}^{\mathrm{N}}$ & $v \tilde{a} \tilde{a}$ & $\begin{array}{l}\text { Consonne orale } \\
\text { + voyelle orale }\end{array}$ & $\mathrm{CV}$ & $\begin{array}{l}\text { papa a } \\
\text { diri a }\end{array}$ \\
\hline $\begin{array}{l}\text { Consonne orale } \\
\text { + voyelle orale } \\
\text { + consonne } \\
\text { nasale }\end{array}$ & $\mathrm{CVC}^{\mathrm{N}}$ & dam nã & $\begin{array}{l}\text { Consonne orale } \\
+ \text { voyelle orale } \\
+ \text { consonne } \\
\text { orale }\end{array}$ & CVC & makak la \\
\hline $\begin{array}{l}\text { Consonne } \\
\text { nasale +voyelle } \\
\text { haute }^{5}\end{array}$ & $\mathrm{C}^{\mathrm{N}} \mathrm{V}_{\text {(+haute) }}$ & зепи а̃ & $\begin{array}{l}\text { Consonne } \\
\text { nasale +voyelle } \\
\text { moins haute }\end{array}$ & $\begin{array}{l}\mathrm{C}^{\mathrm{N}} \mathrm{V}_{(-} \\
\text {haute) }\end{array}$ & $\begin{array}{l}\text { lame a } \\
\text { anana a }\end{array}$ \\
\hline $\begin{array}{l}\text { Consonne } \\
\text { orale+ voyelle } \\
\text { nasale+ } \\
\text { consonne orale }\end{array}$ & $\mathrm{CV}^{\mathrm{N}} \mathrm{C}$ & bãk la & $\begin{array}{l}\text { Consonne } \\
\text { nasale }+ \\
\text { voyelle orale + } \\
\text { consonne } \\
\text { nasale }\end{array}$ & $\mathrm{C}^{\mathrm{N}} \mathrm{VC}$ & klinik la \\
\hline
\end{tabular}

Tableau 6.- Contextes nasals et non nasals 


\subsection{Analyse et résultats}

Afin de trouver des réponses à ces questions, nous avons recruté des locuteurs venus de deux régions géographiques différentes d'Haïti. Un groupe comprenait des résidents de la région urbaine de la commune de Carrefour - l'une des plus grandes municipalités résidentielles de la région de Port-au-Prince -, et l'autre des résidents de Morne-à-Chandelle, l'une des sections rurales de cette même commune. Morne-à-Chandelle se situe à environ 18 kilomètres de la région urbaine de Carrefour et à une vingtaine kilomètres de Port-au-Prince. Douze locuteurs, tous hommes (6 urbains et 6 ruraux) ont été sélectionnés en fonction de leur localité, de leur niveau de scolarité et de leur âge.

Un autre facteur social que nous avons aussi pris en compte au cours de la recherche de terrain est la mobilité. Par exemple, nous avions remarqué que les locuteurs venant de Morne-à-Chandelle avaient eu beaucoup plus de contacts avec la région métropolitaine de Port-au-Prince que leurs parents et grands-parents. Certains d'entre eux continuaient à se déplacer fréquemment du village à la ville pour se rendre à l'école, au travail, etc. Ces jeunes locuteurs (identifiés dans l'étude comme étant des ruraux-mobiles) se sont classés dans un groupe intermédiaire parce qu'ils étaient en contact permanent non seulement avec leurs parents vivant à la campagne, mais aussi avec leurs amis citadins. Après le classement des sujets, deux heures d'interviews ont été menés individuellement avec chaque participant. Voici un extrait du corpus:

\section{(3a) Jeune locuteur bilingue résidant à Carrefour (citadin)}

... Nan boutik lan, li te gon ti boutik tou. Li mete Zoun nan boutik lan... chita... lè $m$ di $w$ nan boutik lan, kounye a la ap... l ap... konfòme de tout bagay... ap gen odèu nan men ni...

Dans la boutique, elle en avait une aussi... Elle a placé Zoun dans la boutique... assise... comme je te le dis, maintenant, elle s'occupait de tout... elle en avait de l'odeur à ses mains ${ }^{6}$

(3b) Locuteur unilingue, âgé et résident de Morne-à-Chandelle (campagne)

Pou mete pitit lan lekòl yo menm tou, fòk yo la avèk pitit la pou yo ka èd pitit lan... pou yo ka èd pitit la

Afin de mettre l'enfant à l'école, eux-mêmes, faut qu'ils soient (les parents) là avec l'enfant pour aider l'enfant... pour pouvoir aider l'enfant

D'après les résultats de ces entretiens, la nasalisation en contexte non nasal est un phénomène qui s'observe chez des locuteurs résidant en ville ou à la campagne, et ceci, indépendamment de leur niveau d'éducation et de leur tranche d'âge. L'exemple (3) montre que le déterminant est nasalisé dans les deux énoncés. Cependant, le locuteur le plus âgé (senior) nasalise beaucoup moins que son jeune homologue (junior). À noter aussi que le l'exemple (3b) relève de ce que Clairis (1991) décrit, lorsqu'il parle de fluctuation, c'est-àdire le phénomène de variation pour un même morphème chez un même locuteur.

L'observation faite précédemment correspond aux résultats présentés dans les graphiques ci-dessous (i.e. 1A-B).

6. Avoir de l'odeur à ses mains fait référence à l'odeur de l'argent, à la circulation de l'argent de mains en mains, particulièrement dans le domaine des affaires. 

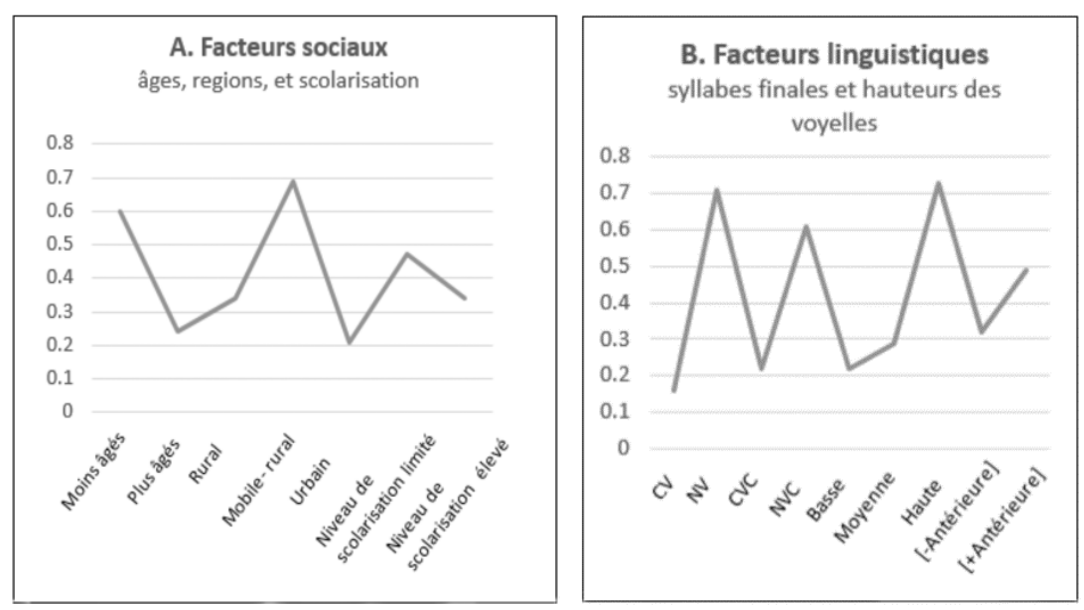

Graphique 1.- Facteurs sociaux et linguistiques favorisant la nasalisation de la ${ }^{7}$

Nous constatons aisément que le taux de fréquence de nasalisation est plus élevé chez les deux groupes de jeunes urbains et ruraux-mobiles. Mais dans l'ensemble, c'est le groupe de locuteurs ruraux-urbains qui nasalisent le plus avec un taux de 0,69 en moyenne par rapport à 0,34 chez leurs homologues ruraux plus âgés. Les jeunes locuteurs urbains ont aussi produit un taux plus élevé (moyenne 0,60) par rapport aux locuteurs urbains les plus âgés (moyenne 0,24$)$ combinés $(0,21$ en moyenne). Soulignons que la majorité des ruraux-mobiles constituent un groupe intermédiaire et qui est en contact direct avec les deux autres groupes de locuteurs (i.e. ruraux et urbains). Dès lors, nous formulons l'hypothèse que ce sont eux-mêmes qui sont responsables de la propagation du changement linguistique de la ville vers la campagne.

Le niveau de scolarisation ne nous paraissait pas être une variable significative. S'agissant des facteurs linguistiques (voir graphique 1B), un lien entre la nasalisation et deux variables a été observé. La première variable est la présence d'une consonne nasale à l'attaque suivie d'une voyelle: $\left(\mathrm{C}^{\mathrm{N}} \mathrm{V}\right)$ : jenou 'genou', lame 'l'armée'). La deuxième est la présence d'une voyelle haute au niveau du noyau du mot précédant le déterminant (par exemple, pitit 'l'enfant', diri 'riz').

Entre les allomorphes nasals [lã] et [ã] on a constaté un taux de fréquence d'occurrence en moyenne de $73 \%$ pour les syllabes finales contenant des voyelles hautes $/ \mathrm{i} /$ et $/ \mathrm{u} /$ (ex. pitimi, zanmi), contre $29 \%$ pour les voyelles mi- fermées $/ \mathrm{e} /, / \varepsilon /, / \mathrm{J} /$, et $/ \mathrm{o} /$ (ex. ane, vòlò, latè) et contre une fréquence moyenne de $22 \%$ lorsque les voyelles basses apparaissent avec une consonne finale (ex. alamanak, patat). Cependant, nous n'avons pas remarqué de cas d'occurrences de nasalisation avec la voyelle [a] dans les mots avec des syllabes finales ouvertes comme. En résumé, ceci indique qu'une syllabe finale de type $\mathrm{C}^{\mathrm{N}} \mathrm{V}$ ou $\mathrm{CV}$ a une plus forte chance d'attirer la nasalisation avec les voyelles hautes $/ \mathrm{i} / \mathrm{et} / \mathrm{u} / \mathrm{qu}$ 'avec $/ \mathrm{e} /, / \mathrm{o} /$ or $/ \mathrm{a} /$. Soit le tableau suivant :

7. Ces résultats sont repris dans le troisième chapitre de notre thèse de doctorat, en cours. 


\begin{tabular}{|l|c|l|c|}
\hline Variables & $\begin{array}{l}\text { Moyenn } \\
\mathrm{e}\end{array}$ & Facteurs sociaux & $\begin{array}{l}\text { Moyenn } \\
\mathrm{e}\end{array}$ \\
\hline $\mathrm{CV}$ & 0.16 & Jeunes & 0.60 \\
\hline $\mathrm{C}^{\mathrm{N} V}$ & 0.71 & Plus agés & 0.24 \\
\hline $\mathrm{CVC}$ & 0.22 & Rural & 0.34 \\
\hline $\mathrm{C}^{\mathrm{N} V C}$ & 0.61 & Rural-mobile & 0.69 \\
\hline Voyelle basse & 0.22 & Urbain & 0.21 \\
\hline $\begin{array}{l}\text { Voyelle mi- } \\
\text { fermée }\end{array}$ & 0.29 & Niveau de scolarisation & 0.47 \\
\hline $\begin{array}{l}\text { Voyelle haute } \\
\text { [-antérieure] }\end{array}$ & 0.73 & $\begin{array}{l}\text { Niveau de scolarisation } \\
\text { avancée }\end{array}$ & 0.34 \\
\hline [+antérieure] & 0.32 & & \\
\hline
\end{tabular}

Tableau 7.- Facteurs sociolinguistiques favorisant la nasalisation de la (Tézil 2014)

En comparant l'effet de la nature de la syllabe sur la nasalisation, il a été constaté que les syllabes qui contiennent une consonne nasale à l'attaque (i.e. $\mathrm{C}^{\mathrm{N} V}$ et $\mathrm{C}^{\mathrm{N}} \mathrm{VC}$ ) avaient une plus forte chance d'attirer la nasalisation que celles qui comprenaient une consonne orale à l'attaque (i.e. CV et CVC). Par exemple, les morphèmes nasals apparaissent avec une fréquence de $71 \%$ après le mot ane 'année', contre $16 \%$ avec le mot pate 'pâté'. Donc étant donné que les morphèmes nasals du déterminant en $\mathrm{CH}$ sont fortement influencés par deux contextes, c'est-à-dire $/ \mathrm{i} /$ et $/ \mathrm{u} /$ et la présence d'une consonne nasale $\left(\mathrm{C}^{\mathrm{N}}\right)$ à l'attaque, il nous parait opportun de soutenir qu'après les mots jenou, zanmi, lanmou, la possibilité d'avoir le déterminant nasalisé est certaine puisque les voyelles hautes sont plus sensibles à la nasalisation. Les mots contenant une syllabe $\left(\mathrm{C}^{\mathrm{N}} \mathrm{V}_{\text {(+haute) }}\right)$ auront une plus forte probabilité d'attirer les morphèmes nasals [ã] et [lã], tandis qu'une syllabe de structure $\left(\mathrm{C}^{\mathrm{N}} \mathrm{V}_{(+ \text {basse }}\right)$ aura une probabilité moins forte à provoquer la nasalisation. La syllabe ayant la moindre influence sur la nasalisation est celle qui comprend une consonne orale et une voyelle basse $\left(\mathrm{CV}_{(+\mathrm{basse})}\right.$. Voici un résumé de la trajectoire de la nasalisation de la en contexte non nasal :

(4) Trajectoire de la du contexte nasal au contexte non nasal

Structure de la syllabe finale: $\mathrm{C}^{\mathrm{N}} \mathrm{V}_{\text {(+haute }}>\mathrm{C}^{\mathrm{N}} \mathrm{V}_{\text {(+basse) }}>\mathrm{CV}_{\text {(+haute) }}>\mathrm{CV}_{\text {(+basse) }}$

Exemple : jenou lame diri papa

Nous avons exclu les syllabes contenant des consonnes finales (i.e. position de coda) dans la trajectoire, car nos données ne nous ont pas permis de confirmer l'effet des consonnes finales sur la nasalisation, comme Valdman l'avait prédit (1991). Nous n'avons pas trouvé de différences significatives entre les syllabes $\mathrm{C}^{\mathrm{N}} \mathrm{VC}$ et $\mathrm{C}^{\mathrm{N} V}$ ou entre les syllabes $\mathrm{CVC}$ et $\mathrm{CV}$. La différence de hauteur vocalique dans les syllabes ouvertes (i.e. $C V$ et $C^{N} V$ ) nous semble être le facteur déterminant dans la nasalisation en contexte non nasal du déterminant en $\mathrm{CH}$. Toutefois, nos données ne nous ont pas permis de vérifier un effet significatif de la voyelle sur le plan antérieur/postérieur.

\subsection{Nasalisation et qualité des voyelles}

Le lien entre la qualité des voyelles et la nasalité a été affirmé dans plusieurs études phonétiques (Rochet \& Rochet 1991, Dow 2014). Par example, Dow (2014 : 199) a constaté qu'en français les voyelles hautes étaient en moyenne $50 \%$ nasales dans les séquences voyelle nasales $(\mathrm{VN})$, bien que le français soit souvent décrit comme une langue dont la nasalisation régressive (ex. / $/ \mathrm{f}^{\mathrm{n}}$ /

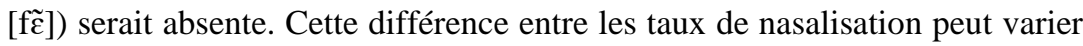


d'une voyelle à l'autre et d'une langue à l'autre. Le même auteur a, par exemple, montré que les taux de nasalisation des voyelles hautes du français et du picard étaient plus élevés que ceux des voyelles moyennes et basses. Le français avait de faibles taux de nasalisation sur les voyelles moyennes et basses, alors qu'ils étaient plus élevés, en picard, en particulier sur la voyelle la/.

Dans la langue Chipewyan, par exemple, les voyelles $[e, \varepsilon]$ alternent avec la voyelle nasale haute [ĩ] (Beddor 1983). Des études approfondies sur l'articulation des sons nasals du français indiquent aussi que le phonème $/ \tilde{\varepsilon} /$ est plus bas et plus postérieur que $/ \varepsilon /$, alors que $/ \tilde{\delta} /$ est plus arrondi et plus postérieur que /o/ (Brichler-Labaeye 1970, Dow 2014). Considérant ces faits, nous partageons la position de Dow et de Beddor sur le fait que la nasalisation d'un phonème oral est un processus qui n'implique pas seulement l'ajout d'un trait de nasalité mais aussi la présence d'autres mécanismes articulatoires qui peuvent même entrainer la modification de la hauteur de la voyelle. Et ce n'est qu'à travers des études approfondies et détaillées au niveau articulatoire (et même phonétique et acoustique) que l'on parvient à observer ce changement.

\section{La nasalisation du déterminant la en contexte non nasal et l'analogie}

La notion d'analogie, popularisée par les néogrammairiens, a été, rappelonsle, utilisée pour rendre compte de la naissance et du changement des formes grammaticales. Saussure (1916) la définit à partir du changement phonétique et note ainsi qu'elle n'implique pas de «transformation du signifiant affecté par le changement, mais création d'un second signifiant pour le même signifié »., Il s'agit, si nous insistons davantage, d'une relation entre un modèle et son imitation. Si nous revenons aux exemples du tableau $2 \mathrm{k}-1$ (jenou an 'le jenou' vs lame a 'l'armée'), nous remarquerons que ces mots ont une structure syllabique similaire en final de mot: consonne nasale +voyelle $\left(\mathrm{C}^{\mathrm{N}}\right)$. Même si le modèle de formation du phonème nasal permet de nasaliser un phonème oral placé en contexte non nasal, la naissance du phonème nasalisé n'élimine pas le phonème oral pour autant, autrement dit, l'emploi de an au lieu de $a$ après diri, mache, kaye, fakilte, bisiklèt, radyo... dans des exemples comme diri an, mache an, kaye an, fakilte an, radyo an, bisifklèt lan n'annule pas les constructions diri a, mache a, kaye a, fakilte a, bisiklèt a et radyo a.Toutefois, aucun locuteur du $\mathrm{CH}$ ne va placer l'allomorphe [a] après les mots ayant une voyelle haute: jenou, pitimi, lanmou. 
(5) Représentation des modèles et de leur imitation

Lexique : NV (ex. lanmou, pitimi, ane, jumu, zanmi, lame, lanmè, diri, peyi,

dra, etc.)

Déterminant : Morphèmes possibles : [ã], [a]

Modèle : $\mathrm{NV}_{(+ \text {haute) }}$ lãmu, pitimi, jumu, zanmi $\rightarrow$ [ã] (Pas de variation possible)

$\mathrm{NV}_{(+ \text {basse) }}$ kanna, anana $\longrightarrow$ [a] (pas de variation possible)

Imitation : $\mathrm{CV}_{(+ \text {haute) }}$ diri, peyi, labou $\longrightarrow$ [ã] (variation possible avec [a])

$\mathrm{CV}_{\text {(+basse) }}$ papa, kaka, dra $\longrightarrow$ [a] (pas de variation possible)

Sans l'aide d'un modèle spécifique, un apprenant aurait du mal à utiliser le déterminant en $\mathrm{CH}$, il confondrait l'emploi [a] et [ã] après les mots jenou et lame. Nous supposons, de ce point de vue, que l'apprenant acquiert un modèle qui lui signale qu'avec les mots contenant une syllabe composée d'une consonne nasale et d'une voyelle (NV) c'est le morphème nasal [ã] qui suit la voyelle haute (i.e. [i], [u]). L'ayant donc acquis, l'enfant pourra généraliser ce modèle et l'appliquer à d'autres mots qui ont cette même caractéristique phonétique tels que : pitimi, lanmou, zanmi, etc.

En réalité, ce modèle n'empêche pas de faire des innovations, autrement dit, de s'éloigner de ce qu'un certain académisme pourrait appeler la norme, pour produire d'autres choses par imitation du modèle préexistant (ex. diri an/a mais papa a).

\subsection{Nasalisation en contexte non nasal: un fait de l'analogie}

$\mathrm{Au}$ cas où une nouvelle structure (syntaxique, morphologique, morphophonologique et même phonétique) aurait surgi, le modèle et la forme de base ne changent pas. Comme Peyraube (2002 : 49) l'a à l'instar de Saussure (1916) mentionné : «L'analogie modifie la structure de surface, mais ne modifie pas la structure sous-jacente.» L'application du modèle de formation des allomorphes nasals [ã] et [lã] aux segments oraux est un changement morpho-phonologique qui ne contrarie pas le fonctionnement interne du système de détermination du CH. Que l'allomorphe se nasalise ou pas, il retient la même fonction syntaxique dans la langue. Il s'agit par ailleurs d'une dynamique de la langue que l'on observe dans d'autres catégories grammaticales.

Nous observons également que le phénomène de nasalisation en contexte non nasal ne se produit pas qu'avec les groupes nominaux (ex. tab la), mais dans d'autres contextes où la forme deictique la généralement apparait en $\mathrm{CH}$. Voici quelques extraits de notre corpus illustrant les occurrences de nasalisation de la au cours d'un entretien réalisé avec une étudiante en droit de la commune de Carrefour.

(6) Après les syllabes NV et NVC

pou fanmi an

nan klinik lan

(7) Après les syllabes CVC

Ale legliz lan... Kèk maten... Bon... kèk maten $m$ leuve boneu male. Mwen vwaye anndan peyi an. Non, se pa justis lan ki gen pwoblèm

(8) Avec les syllabes CV

Te gen twa [3] timoun nan lakou an

Son seri deu moun ki t ap fè bru an kouri pou te ka pluzoumwen piye moun yo tou.

(9) Avec des voyelles non hautes

Pandan tout ane an tou $m$ te fè pluzyeur spetakl

Kèk fwa m konn anvi swiv kèk kou mwen ale nan fakulte an dirèkteuman

(10) Extension de la nasalisation au-delà des syntagmes nominaux

Mwen renmen jan vil lan ye an li menm

Donk leu moun nan ap imite sa $k$ soti an konsa 
Katreuven sis [86] juska jodi an la, yo toujou la

Li pase nan koulwa lakay li an;

(11) Nasalisation avec le [r] postvocalique

Se kraze l net, kraze ansyen presbitèr lan

$M$ wè l nan karfur lan $k$ ap vini

(12) Occurrences de nasalisation en divers contextes

E keu lè l tire polisye an, skandal lan pou fanmi an.

Les exemples ci-dessus montrent qu'il existe en $\mathrm{CH}$ une extension du phénomène de nasalisation qui affecte non seulement les syntagmes nominaux mais les structures phrastiques, les adverbes, et dans presque tous les contextes dans lesquels le morphème la est utilisé, autrement dit, qu'il soit pris comme déterminant ou comme déictique. Les cas d'occurrences de nasalisation avec les noms sont illustrés aux exemples (6), (7), (8) et (9) (ex. legliz lan 'l'église', peyi an 'le pays'). Les morphèmes nasals du déterminant apparaissent avec les voyelles hautes et les voyelles moyennes ou mifermées (ex. ane an 'l'année'). En (12), nous constatons que la nasalisation affecte tous les noms en dépit des différences de hauteur vocalique. Il faudra, pour cela, rappeler que la nasalisation en contexte non nasal est : 1) une variante du modèle 2), un changement qui n'affecte pas toutes les voyelles au même niveau, 3) sa trajectoire n'est pas catégorique mais plutôt une échelle dont les voyelles hautes et basses (i.e. les deux modèles) constituent les deux extrémités.

\subsection{Conceptualisation du changement et de sa trajectoire}

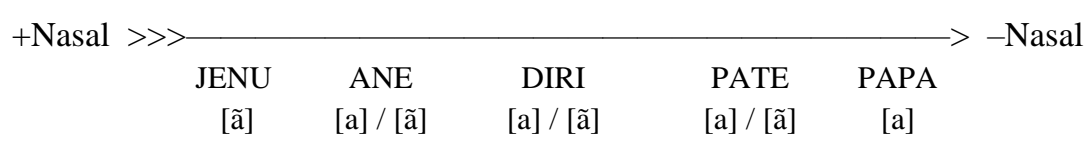

Même dans les contextes non nasals (ex. diri 'riz' vs papa 'père'), les voyelles hautes ont une plus forte chance d'attirer la nasalisation par rapport à celles qui sont moins hautes (Tableau 6). Cela explique pourquoi les mots ayant une voyelle haute comme /i/ et /u/ à la fin sont placés plus près du modèle d'à gauche (i.e. jenou 'genou'), alors que les mots contenant des voyelles moins hautes comme /e/, /o/ se situent plus à proximité du modèle d'à droite (ex. papa 'père').

Cette échelle est mieux saisie et adaptée dans une approche quantitative car les analyses statistiques sont minutieusement calculées et comparées en termes de probabilité et non d'après une approche catégorique. Nous n'ignorons pas que la présence d'une consonne finale dans une syllabe finale de type CVC peut augmenter la chance d'attirer les morphèmes nasals même avec une voyelle basse (ex. tabak la/lan 'le tabac').

\subsection{Variation et compétition en analogie}

L'analogie aide à réguler et à mieux exploiter la variation linguistique. En usage, les formes de la langue varient en raison de facteurs sociaux, psychologiques, linguistiques, et autres. En anglais, par exemple, certains locuteurs utilisent dived pour marquer le passé du verbe dive 'plonger', alors que d'autres emploient dove. En utilisant la forme dived pour indiquer que le procès dive est au passé, cette catégorie de locuteurs suit le modèle de formation du passé régulier qui se forme par l'ajout du suffixe -ed au radical (ex. study 'étudier' > studied). Consciemment ou pas, ces derniers innovent selon un modèle préexistant. L'autre catégorie opte plutôt pour la reproduction du modèle de formation du passé irrégulier des verbes par l'emploi d'un modèle comme celui appliqué pour le cas du verbe drive 'conduire' dont le temps du passé est drove. 
La différence entre les morphèmes a été observée dans la grammaire générative, particulièrement dans les études d'Anderson (1968) et de Kiparsky (1973). La question qu'ils se sont posée est de savoir comment trancher lorsque le lexique d'une langue comme l'anglais, par exemple, contient le morphème go 'aller', -ed (spécifié pour le temps du passé) et went qui est aussi spécifié pour le temps passé du verbe go. Etant donné que le morphème went $\mathrm{a}$ un plus grand nombre de traits qui sont fusionnés en une seule forme avec plus de spécificité, c'est lui qui est le favori de la compétition (indiqué par le symbole $\longrightarrow$ ).

(13) Compétition entre les passés régulier et irrégulier (Ackema \& Neeleman 2005: 287)

a. [GO PAST] (morphosyntax)

b. [/go//ed/] (morphonology)

b'. $\rightarrow$ [/went/] (morphophonology)

Le choix entre les morphèmes favoris et non favoris est fait à partir des contraintes linguistiques et des principes régulateurs appelés en anglais Elsewhere Principle (Kiparky 1973) stipulant qu'en linguistique, l'application d'une règle ou d'une opération spécifique peut annuler ou passer outre l'application d'une règle plus générale.

Nous constatons couramment chez certains enfants et même chez certains adultes anglophones, l'attachement du suffixe du passé régulier -ed au morphème went dans des énoncés comme celui-ci: "He could have have wented" au lieu de "He could have gone" 'Il aurait pu y aller'. Donc, si la création de la forme irrégulière went est due au changement linguistique qui avait affecté la langue anglaise, l'analogie permet aux locuteurs de continuer à créer de nouvelles formes régulières, se servant parfois même des formes irrégulières à la base. 


\subsection{L'acquisition de la nasalisation en contexte nasal: le rôle de I'analogie}

Celui qui apprend le $\mathrm{CH}$ fait face à ce changement linguistique en cours. Mais comme il est impossible de mémoriser un nombre infini d'occurrences et de variations, l'acquisition des règles de modèle paraît plus plausible et judicieuse. Tout locuteur haïtien considérerait, par exemple, les syntagmes : « jenou a, lanmou a » comme erronés par rapport aux syntagmes «jenou an, lanmou an ». De même, la majorité d'entre eux seraient d'accord qu'un enfant qui dirait «papa an, anana an » au lieu de «papa a et anana $a$ » aurait besoin d'être corrigé. Comme indiqué au tableau 8, la présence du morphème nasal est obligatoire au modèle $\mathrm{A}$ à gauche, alors qu'à droite (i.e. modèle B) c'est le morphème oral qui est obligatoire. Au milieu, il existe de la variation entre les deux modèles, et qui est devenue de plus en plus acceptable. Lorsque certaines formes en compétition sont acceptables, le locuteur en devient de moins en moins conscient car le changement (i.e. la nasalisation en contexte oral) est moins stéréotypé (Labov 2001).

\begin{tabular}{|l|l|l|l|l|}
\hline Modèle A & $\begin{array}{l}\text { Contextes } \\
\text { cibles 1 }\end{array}$ & $\begin{array}{l}\text { Contextes cibles } \\
2\end{array}$ & $\begin{array}{l}\text { Contextes cibles } \\
3\end{array}$ & Modèle B \\
\hline jenou an & lame a/an & diri a/an & tab la/lan & kaka a \\
\hline pitimi an & kenèp la/lan & lakou a/an & bebe a/an & papa a \\
\hline zanmi an & kanè a/an & bouk la/lan & nap la/lan & anana a \\
\hline fanmi an & zanno a/an & kounouk la/lan & patat la/lan & zaboka a \\
\hline joumou an & klinik la/an & tòti a/an & pap la/lan & galata a \\
\hline lanmou an & nòt la/lan & mizik la/lan & almanak la/lan & pwa a \\
\hline
\end{tabular}

Tableau 8.- La nasalisation de la en contexte non nasal et l'acquisition

Dans les syllabes ouvertes, ce n'est pas forcément la présence de la consonne nasale $\left(\mathrm{C}^{\mathrm{N}}\right)$ qui détermine la nasalisation mais la hauteur des voyelles, plus précisément lorsqu'elles se trouvent en fin de mots. Sinon le déterminant serait nasalisé après anana «ananas ${ }^{8}$ Partant de ce principe, nous soutenons que les modèles acquis peuvent varier, au regard surtout des données que nous indiquons dans le tableau 8.

\section{Conclusion}

L'utilisation des morphèmes nasals [lã] et [ã] après les segments oraux (ex. tab lan 'la table' et peyi an 'le pays') est un changement linguistique en cours. Nos résultats ainsi que ceux de Valdman (1991) ont révélé que le choix entre les syntagmes peyi a et peyi an 'le pays' n'est pas le fruit du hasard, mais un choix motivé et utile (même si certains locuteurs n'en sont pas toujours conscients), déterminé par des facteurs sociolinguistiques, stylistiques et cognitifs. Valdman (1991) a remarqué que les jeunes haïtiens bilingues vivant dans la région métropolitaine de Port-au-Prince avait tendance à nasaliser plus fréquemment en contexte non nasal que les locuteurs plus âgés. Nous sommes encore allés plus loin jusqu'à démontrer que ce changement observé est déterminé à partir du modèle de formation des mots par analogie (Saussure 1916). Toutefois, même si la nasalisation du déterminant s'est généralisée dans presque tous les contextes (i.e. voyelle haute, moyenne et même avec les voyelles basses dans les syllabe fermées), il y existe encore une certaine résistance à la nasalisation au niveau des

${ }^{8}$ Il est possible que la voyelle se nasalise par assimilation progressive avec la consonne nasale de l'attaque. Toutefois, nous supposons que les voyelles hautes attirent la nasalisation plus facilement car le déterminant ne se nasalise jamais lorsqu'il est suivi de [a], et même avec $\mathrm{C}^{\mathrm{N}}$. 
voyelles basses, particulièrement lorsque celles-ci se trouvent dans une syllabe ouverte (ex. papa a/*an 'le père', anana a/*an 'l'ananas').

A propos de l'acquisition, il a été montré que l'enfant acquiert les modèles avant de les généraliser. La généralisation des formes dépendra de plusieurs facteurs (social, linguistique, stylistique, etc.). L'enfant qui apprend à parler intègre aussi les normes sociales ainsi que leur influence sur la langue. Ceci explique pourquoi il afficherait une certaine préférence pour une forme au détriment d'une autre. Notre analyse sous-entend que les variations linguistiques ne sont pas toujours des faits d'exception ou de violation de la règle générale de la langue, puisqu'elles se manifestent constamment et résultent des faits dynamiques de la langue. Les contraintes linguistiques (Prince \& Smolensky 1993), quant à elles, ne changent pas lorsqu'il y a variation, mais se réarrangent et se reclassifient. Lorsqu'il y a variation entre les morphèmes nasals et oraux, ces contraintes sont structurées de manière à ce que la forme favorite soit gagnante.

L'emploi des morphèmes nasals [ã] et [1ã] en contexte non nasal suit et imite deux modèles : celui dont la syllabe finale contient une consonne nasale et une voyelle haute $\left(\mathrm{C}^{\mathrm{N}} \mathrm{V}_{\text {(+haute) }}\right.$ : jenou an 'le jenou') et celui dont la syllabe finale comprend une consonne nasale et une voyelle basse $\left(\mathrm{C}^{\mathrm{N}} \mathrm{V}_{(+\mathrm{basse})}\right)$ : anana a 'l'ananas'). Cela passe par l'application du modèle de formation par analogie qui met en exergue la capacité cognitive des locuteurs. Dans les syllabes ouvertes, les voyelles basses résistent au changement linguistique. Est-ce pourquoi jusqu'à présent les mots anana, papa, kaka, ne sont jamais suivis de [(1)ã] en $\mathrm{CH}$. Cette résistance des voyelles basses s'explique en raison du phénomène de coarticulation impliquant deux morphèmes identiques qui se fusionnent en une voyelle longue. Par exemple, le syntagme papa a 'le père' se représente en phonologie ainsi : /papa la/ qui devient [papaa] et qui se produit phonétiquement avec une voyelle allongée : [papa:]. Ceci explique pourquoi, nous soutenons que le fusionnement des deux voyelles identiques empêche la nasalisation de s'étendre au niveau des voyelles basses.

\section{Ouvrages cités}

AcKema Peter and NeELman Ad, 2005, "Word-Formation in Optimality Theory", in Pavol Stekauer and Rochelle Lieber, Handbook of Word Formation, Dordrecht, Springer, p. 285-313.

AlBright Adam, 2008, "Explaining Universal Tendencies and Language Particulars in Analogical Change", in Jeff Good (ed.), Language Universals and Language Change, Oxford, Oxford University Press, p. 144-184.

ANDERSON Stephen R., 1968, West Scandinavian Vowel System and the Ordering of Phonological Rules, Thèse de doctorat, MIT.

Anttila Raimo, 1977, Analogy, The Hague, Mouton.

ARLOTTO Anthony, 1972, Introduction to Historical Linguistics, Lanham, University Press of America.

BEDDOR Patrice Speeter,1983, Phonological and Phonetic Effects of Nasalization on Vowel Height, Bloomington (IN), Indiana University Linguistics Club.

BERNABE Jean, 1983, Fondal-natal: grammaire basilectale approche des créoles guadeloupéen et martiniquais: approche sociolittéraire, sociolinguistique et syntaxique (Vol. 1), Paris, L'Harmattan.

Bybee Joan L., 1985, Morphology: A Study of the Relation Between Meaning and Form, Amsterdam, Benjamins.

ByBEE Joan L.,, 2008, "Formal universals as emergent phenomena", in Jeff Good (ed.), Linguistic Universals and Language Change, Oxford, Oxford University Press, p. 108-124.

CADELY Jean-Robert, 1994, Aspects de la phonologie du créole haïtien, Thèse de doctorat de l'Université du Quebec à Montréal. 
CAdely Jean-Robert, 1996, "Nasality in Haitian Creole: The Case of the Determiner", communication au $11^{\mathrm{e}}$ congrès biennal de la Society for Caribbean Linguistics, Saint-Martin.

CADELY Jean-Robert, 2002, «Le statut des voyelles nasales en créole haïtien », Lingua 112 (6), p. 435-464.

CAmpbell Lyle, 1998, Historical Linguistics. An Introduction. Edinburgh, University Press.

CLAIRIS, Christos. 1991, «Identification et typologie des fluctuations ». BSL Tome LXXXVI. 19-35.

DAMOISEAU Robert, 1984, Éléments de grammaire du créole martiniquais, Fort-deFrance, Hatier-Antilles.

DegrafF Michel, 2007, "Kreyòl Ayisyen, or Haitian Creole (Creole French)", in John Holm and Peter L. Patrick (eds.), Comparative Creole Syntax: Parallel Outlines of 18 Creole Grammars, London, Battlebridge, p. 101-126.

DeJean Yves, 1980, Comment écrire le créole d'Haïti, Outremont (Québec), Collectif Paroles.

Dow Michael, 2014, Contrast and Markedness Among Nasal(ized) Vowels : A Phonetic Phonological Study of French and Vimeu Picard. Thèse de doctorat, Indiana University.

FAINE Jules, 1937, Philologie créole: études historiques et étymologiques sur la langue créole d'Haiti, Port-au-Prince, Imprimerie de l'État.

GENTNER Dedra and JEZIORSKI Michael, 1989, "Historical Shifts in the Use of Analogy in Science", in B. Gholson, W. R. Shadish, R. A. Beimeyer and A. Houts (Eds.), The Psychology of Science: Contributions to Metascience, Cambridge, Cambridge University Press, p. 296-325.

GENTNER Dedra and Jeziorski Michael, 1993, "The Shift from Metaphor to Analogy in Western Science", in A. Ortony (Ed.), Metaphor and Thought, $2^{\text {nd }}$ ed., Cambridge, Cambridge University Press, p. 447-480.

Hall Robert A. Jr., 1953, Haitian Creole: Grammar, Texts, Vocabulary, Philadelphia, American Folklore Society, Memoirs 43.

HasPelmath Martin, 2008, "Creating Economical Morphosyntactic Patterns in Language Change", in Jeff Good (ed.), Language Universals and Language Change, Oxford, Oxford University Press, p. 185-214.

PhILADElPHIA Martin and SiMS Andrea D., 2010, Understanding Morphology, Oxford, London and New York, Oxford University Press.

Hock Hans Henrich, 1991, Principles of Historical Linguistics, $2^{\text {nd }}$ ed., Berlin, De Gruyter Mouton.

HoGg Richard M.,1979, “Analogy and Phonology”, Journal of Linguistics, vol. 15, $\mathrm{n}^{\circ} 1, \mathrm{p} .55-85$.

HuAlDE José Ignacio, 2006, "Analogy and Other Types of Non-Phonetic Change in Bizkaian Basque", in J. Lakarra and J. I. Hualde (eds.), Studies in Basque and Historical Linguistics in Memory of R. L. Trask. R. L. Trasken oroitzapenetan ikerketak euskalaritza eta hizkuntzalaritza historikoaz, ASJU XL 1-2

JEAN-BAPTISTE Rozevel, 1992, Étude syntactico-sémantique des zones de prédication et de détermination en créole haütien: essai sur quelques micro-opérations. Thèse de doctorat, Université Paris V.

JoSEPH Yves, 1984, «Les déterminant définis et indéfinis en haïtien : une approche générative », Conjonctions [Port-au-Prince], p.161-162.

KIPARSKY Paul, 1973, "Elswhere in Phonology", in S. Anderson and P. Kiparsky (eds.), A Festschrift for Morris Halle, New York, Holt, Rinehart and Winston, p. 93-106.

Labov William, 2001, Principles of Linguistic Change, I: Internal Factors, II: Social Factors, Oxford, Blackwell.

MacMahon April M.S., 1994, Understanding Language Change, Cambridge, Cambridge University Press.

Mortureux Marie-Françoise, 1974, «Analogie "créatrice", formelle et sémantique », dans Louis Guilbert, Langages, $\mathrm{n}^{\circ} 36$, La Néologie lexicale, p. 20-33. 
NiKIEMA Emmanuel, 1999, «De la variation du déterminant /la/ dans les créoles haïtien et saint-lucien», Lingua, $\mathrm{n}^{\circ}$ 107(1), p. 69-93.

O'Grady William, ArChiBALd John, Aronoff Mark and ReES-Miller Janie, 2005, Contemporary Linguistic Analysis: An Introduction (5 $\left.{ }^{\text {th }} \mathrm{ed}.\right)$, Boston, St-Martin's.

PaOlillo John C., 2002, Analyzing Linguistic Variation: Statistical Models and Methods, Stanford (CA), CSLI (Center for the Study of Language and Information).

PeYraube Alain, 2002, «L'évolution des structures grammaticales », Langage, $\mathrm{n}^{\circ} 146$, p. 46-58.

Prince Alan et Smolensky Paul, 1993, Optimality Theory, Constraint Interaction in Generative Grammar, Rutgers (online).

RIPOLL Thierry and Coulon David, 2001, «Le raisonnement par analogie : une analyse descriptive et critique des modèles du mapping ", L'Année psychologique. t. 101, fasc. 2, p. 289-323.

Rochet Anne Putnam and Rochet Bernard L., 1991, "The Effect of Vowel Height on Patterns of Assimilation Nasality in French and English", in Proceedings of the 12th International Congress of Phonetic Sciences, vol. 3, p. 54-57, Aix-en-Provence.

SAUSSURE Ferdinand de, 1916, 1972, Cours de linguistique générale, éd. Tullio De Mauro, Paris, Payot.

SCHIEFFELIN Bambi et Doucet Rachelle Charlier, 1994, "The Real Haitian Creole: Ideology, Metalinguistics, and Orthographic Choice", Am. Ethnol. $\mathrm{n}^{\circ} 21$, p. 176-200.

Sturtevant Edgar H., 1947, An Introduction to Linguistic Science, New Heaven, Yale University Press.

Sylvain Suzanne C., 1936, Le Créole haïtien, morphologie et syntaxe, Wetteren (Belgique) de Meester et Port-au-Prince, chez l'auteure.

Tagliamonte Sali A., 2006, Analysing Sociolinguistic Variation, Cambridge and New York, Cambridge University Press.

TAYLOR Douglas R, 1968, «Le créole de La Dominique », dans A. Martinet (éd.), Le Langage, Paris, Gallimard, «Encyclopédie de la Pléiade».

TÉZIL David (à paraître), A Variationist Study of the Haitian Post-posed Determiner LA in Non-nasalized Contexts. Doctoral Dissertation. Indiana University.

Tinelli Henri, 1981, Creole Phonology, The Hague, Mouton.

TRASK Larry R., 1996, Historical Linguistics, London, Arnold.

TRASK Larry R., 2007, Trask's Historical Linguistics, $2^{\text {nd }}$ ed., revised by Robert McColl Millar, London, Hodder.

VALDMAN Albert, 1978, Le Créole : Structure, statut et origine, Paris, Klincksieck.

VALDMAN Albert, 1991, "Decreolization or Dialect Contact in Haiti?", in Francis Byrnes and Thomas Huebner (eds.), Development and Structures of Creole Languages: Essays in Honor of Derek Bickerton, Amsterdam and Philadelphia, Benjamins, p. 75- 88.

Valdman Albert, 2015, Haitian Creole: Structure, Variation, Status, Origin, Bristol (CT), Equinox.

VALDMAN Albert and IsKRova Iskra, 2003, "A New Look at Nasalization in Haitian Creole", The Phonology and Morphology of Creole Languages, Tübingen, Niemeyer, p. 25-41.

WetZels Willem Leo Marie, 1982, Analogie et lexique : le problème de l'opacité en phonologie générative, Thèse de doctorat, Catholic University Nijmegen. 